\title{
OryzaGenome2.1: Database of Diverse Genotypes in Wild Oryza Species
}

\author{
Hiromi Kajiya-Kanegae ${ }^{1}$, Hajime Ohyanagi ${ }^{2}$, Toshinobu Ebata ${ }^{3}$, Yasuhiro Tanizawa ${ }^{4}$, Akio Onogi ${ }^{5}$, Yuji Sawada ${ }^{6}$, \\ Masami Yokota Hirai ${ }^{6}$, Zi-Xuan Wang ${ }^{7}$, Bin Han ${ }^{7}$, Atsushi Toyoda ${ }^{4}$, Asao Fujiyama ${ }^{4}$, Hiroyoshi Iwata', \\ Katsutoshi Tsuda ${ }^{4}$, Toshiya Suzuki ${ }^{4}$, Misuzu Nosaka-Takahashi ${ }^{4}$, Ken-ichi Nonomura ${ }^{4}$, Yasukazu Nakamura ${ }^{4}$, \\ Shoko Kawamoto ${ }^{4}$, Nori Kurata ${ }^{4}$ and Yutaka Sato ${ }^{4^{*}}$ (D)
}

\begin{abstract}
Background: OryzaGenome (http://viewer.shigen.info/oryzagenome21 detail/index.xhtml), a feature within Oryzabase (https://shigen.nig.ac.jp/rice/oryzabase/), is a genomic database for wild Oryza species that provides comparative and evolutionary genomics approaches for the rice research community.

Results: Here we release OryzaGenome2.1, the first major update of OryzaGenome. The main feature in this version is the inclusion of newly sequenced genotypes and their meta-information, giving a total of 217 accessions of 19 wild Oryza species (O. rufipogon, O. barthii, O. longistaminata, O. meridionalis, O. glumaepatula, O. punctata, O. minuta, $O$. officinalis, $O$. rhizomatis, $O$. eichingeri, $O$. latifolia, $O$. alta, $O$. grandiglumis, $O$. australiensis, $O$. brachyantha, $O$. granulata, O. meyeriana, O. ridleyi, and O. longiglumis). These 19 wild species belong to 9 genome types (AA, BB, CC, $\mathrm{BBCC}, \mathrm{CCDD}, \mathrm{EE}, \mathrm{FF}, \mathrm{GG}$, and $\mathrm{HHJJ})$, representing wide genomic diversity in the genus. Using the genotype information, we analyzed the genome diversity of Oryza species. Other features of OryzaGenome facilitate the use of information on single nucleotide polymorphisms (SNPs) between O. sativa and its wild progenitor O. rufipogon in rice research, including breeding as well as basic science. For example, we provide Variant Call Format (VCF) files for genome-wide SNPs of 33 O. rufipogon accessions against the O. sativa reference genome, IRGSP1.0. In addition, we provide a new SNP Effect Table function, allowing users to identify SNPs or small insertion/deletion polymorphisms in the 33 O. rufipogon accessions and to search for the effect of these polymorphisms on protein function if they reside in the coding region (e.g., are missense or nonsense mutations). Furthermore, the SNP Viewer for 4460. rufipogon accessions was updated by implementing new tracks for possible selective sweep regions and highly mutated regions that were potentially exposed to selective pressures during the process of domestication.

Conclusion: OryzaGenome2.1 focuses on comparative genomic analysis of diverse wild Oryza accessions collected around the world and on the development of resources to speed up the identification of critical trait-related genes, especially from O. rufipogon. It aims to promote the use of genotype information from wild accessions in rice breeding and potential future crop improvements. Diverse genotypes will be a key resource for evolutionary studies in Oryza, including polyploid biology.
\end{abstract}

Keywords: Database, Genome diversity, Oryza, NIG wild Oryza collection, Oryzabase, Oryza rufipogon, Polyploidy

\footnotetext{
* Correspondence: yusato@nig.ac.jp

${ }^{4}$ National Institute of Genetics, Yata 1111, Mishima, Shizuoka 411-8540, Japan

Full list of author information is available at the end of the article
}

\section{Springer Open}

(c) The Author(s). 2021 Open Access This article is licensed under a Creative Commons Attribution 4.0 International License, which permits use, sharing, adaptation, distribution and reproduction in any medium or format, as long as you give appropriate credit to the original author(s) and the source, provide a link to the Creative Commons licence, and indicate if changes were made. The images or other third party material in this article are included in the article's Creative Commons licence, unless indicated otherwise in a credit line to the material. If material is not included in the article's Creative Commons licence and your intended use is not permitted by statutory regulation or exceeds the permitted use, you will need to obtain permission directly from the copyright holder. To view a copy of this licence, visit http://creativecommons.org/licenses/by/4.0/. 


\section{Background}

Rice is an essential food for humankind, playing a critical role in food security. In addition, rice is a well-established model monocot in plant science, enabling advanced genomic breeding approaches for rapid development of improved cultivars. One such approach is genomic introgression from either closely or distantly related wild Oryza species into cultivated rice species, with the aim of exploiting agronomically advantageous traits from diverse wild relatives.

While a massive number of Asian cultivated rice (Oryza sativa L.) genotypes have been made available to the public (Alexandrov et al. 2015) (Wang et al. 2018), few wild Oryza genotypes have been released. To provide an open-access platform for genomic information on highly diverse wild Oryza species, OryzaGenome (Ohyanagi et al. 2016) was launched in 2015. The original version included the basic function of SNP viewer for 446 O. rufipogon, in addition to genotype information of 446 O. rufipogon accessions and 17 Oryza accessions. It also included hyperlinks to phenotypic and habitat information in Oryzabase (Kurata and Yamazaki 2006) to provide a functional genomics approach for the wild Oryza research community.

Here, we release OryzaGenome2.1 (http://viewer. shigen.info/oryzagenome21detail/index.xhtml), a major update. This release features newly sequenced genotypes and their meta-information, for a total of 217 wild Oryza accessions from 19 Oryza species (Table 1). It also provides an intuitive single nucleotide polymorphism (SNP) Effect Table functionality using 33 deep-sequenced $O$. rufipogon accessions in addition to a SNP Viewer with new information tracks for 446 imputed O. rufipogon genotypes. Because the 19 wild species belong to 9 genome types, this release will provide access to the genomic diversity within the genus Oryza for comparative and evolutionary genomics, as well as the potential for further improvement in rice breeding practices.

\section{Materials and Methods}

\section{Reference Genome Information}

We used Os-Nipponbare-Reference-IRGSP-1.0 (O. sativa ssp. japonica 'Nipponbare') as a reference genome sequence (Kawahara et al. 2013). For gene annotations, we used MSU Rice annotations (MSU Rice Genome Annotation Project Team 2015) and RAP-DB annotations (Sakai et al. 2013).

\section{Genomic Data for 217 Oryza Genotypes}

Of the 217 wild Oryza accessions (Table 1, Supp. Table 1), 213 are maintained at the National Institute of Genetics (Nonomura et al. 2010), and the remaining 4 were obtained from IRRI. DNA was extracted from leaves and sequenced as described (Shenton et al. 2020). Short-read Illumina sequencing was performed on an Illumina HiSeq sequencer with paired reads (Illumina, San Diego, CA). Each raw sequence is available via the National Center for Biotechnology Information (NCBI) and DNA Data Bank of Japan (DDBJ) Sequence Read Archive (DRA) system.

\section{K-Mer Analysis}

$K$-mer analysis was conducted using GenomeScope (v1.0.0) following the instruction of the software (Vurture et al. 2017). K-mer frequency was counted using Jellyfish ver. 2.2.6 with the $k$-mer size of 21 and the "cannonical kmers" option (-C) (Marcais and Kingsford 2011). The resultant $k$-mer histogram was subjected to GenomeScope to estimate genomic properties such as genome size, repeat content, and heterozygosity. For several accessions, the model fit of GenomeScope did not converge, possibly because of the low sequencing coverage.

\section{Genomic Data and VCF Files for 33 O. rufipogon Accessions}

Thirty-three O. rufipogon accessions (Table 1, Supp. Table 1) were genotyped at higher coverage (average $19.5 \times)$. To process the resequencing reads from these accessions, we modified the analytical workflow originally developed for the TASUKE+ variant browser of the Rice Annotation Project Database (RAP-DB; https:// rapdb.dna.affrc.go.jp/genome-wide_variations/Analysis_ workflow_for_detection_of_genome-wide_var.html)

(Kumagai et al. 2019). Briefly, paired-end reads preprocessed by Trimmomatic v. 0.38 (Bolger et al. 2014) were

Table 1 List of information released in OyrzaGenome2.1

\begin{tabular}{lll}
\hline & $\mathbf{2 1 7}$ Wild Oryza Genome Sequences & $\mathbf{3 3}$ Deep-sequenced O. rufipogon \\
\hline Number of Accessions & $217^{\text {a }}$ & 33 \\
Species & 19 wild Oryza species & Imputed $\mathbf{4 4 6}$ O. rufipogon \\
Raw NGS data & $\checkmark$ & $\checkmark$ \\
Imputation-free variants & & $\checkmark$ \\
SnpEff-based Variant Lists & $\checkmark$ & $\checkmark$ \\
Imputed variants & & $\checkmark$ \\
\hline
\end{tabular}

Including 33 Deep-sequenced O. rufipogon 
mapped on the IRGSP1.0 reference genome (including organellar and unanchored contig sequences) using the bwa-0.7.17 mem ( $\mathrm{Li}$ and Durbin 2010) algorithm with the default options. PCR duplicates were removed using MarkDuplicates of the Picard package v. 2.18.17 (http:// broadinstitute.github.io/picard). Variants were called by HaplotypeCaller of the Genome Analysis Toolkit (GATK, v. 4.0.11.0) (McKenna et al. 2010). They were filtered using VariantFiltration of GATK with filter expressions of "DP $<2 \|$ QD $<2.0 \|$ FS $>60.0 \| \mathrm{MQ}<$ 40.0 || SOR > 3.0 || MQRankSum < -12.5 || ReadPosRankSum $<-8.0$ " for homozygous variants. Finally, the variants were annotated using SNPeffect v. 4.3 (Cingolani et al. 2012) based on the gene annotation available at RAP-DB (release 26 Nov 2018).

The raw sequence data as well as variant information in Variant Call Format (VCF) files are accessible from the Downloads page (Fig. 1). The analytical pipeline implemented in Common Workflow Language is available from https://github.com/nigyta/rice_reseq.

\section{Genomic Data for $\mathbf{4 4 6}$ Imputed O. rufipogon Variants} Imputed genome data were obtained as follows. Wholegenome information in OryzaGenome v. 1 Variant List (Ohyanagi et al. 2016) (http://viewer.shigen.info/ oryzagenome $/ \mathrm{mapview} / \mathrm{VariantView} \cdot \mathrm{do}$ ?action= Downloads) was downloaded and re-imputed by beagle 4.0 software (Browning and Browning 2007) (https:// faculty.washington.edu/browning/beagle/b4_0.html).
This file contains phased, non-missing genotypes for all non-reference positions and is available from the Downloads page.

\section{System Architecture and Software}

OryzaGenome2.1 is implemented on a UNIX server with CentOS v. 7, with Apache/Tomcat and Apache/GlassFish web servers and a PostgreSQL Database server.

Java and $\mathrm{C}++$ were used as server-side application languages. JavaScript was used to implement client-side rich applications. The JavaScript libraries jQuery (http:// jquery.com), DataTables (https://www.datatables.net/), Magnific Popup (http://dimsemenov.com/plugins/ magnific-popup/), Google chart tools (https://developers. google.com/chart/), Prototype (http://prototypejs.org/), and script.aculo.us (https://script.aculo.us/) were used. Other conventional utilities for UNIX computing were installed on the server as necessary. All the OryzaGenome2.1 resources are stored on the server and are available at http://viewer.shigen.info/oryzagenome21detail/ index.xhtml.

\section{Results and Discussion}

\section{Genotypes and Batch Downloads}

OryzaGenome release 2.1 provides the genotypes of 217 highly diverse accessions from 19 wild Oryza species (average coverage 18x) (Table 1; Supp. Table 1). Among the 217 Oryza accessions, 33 O. rufipogon accessions were genotyped at higher coverage (average 19.5×), and

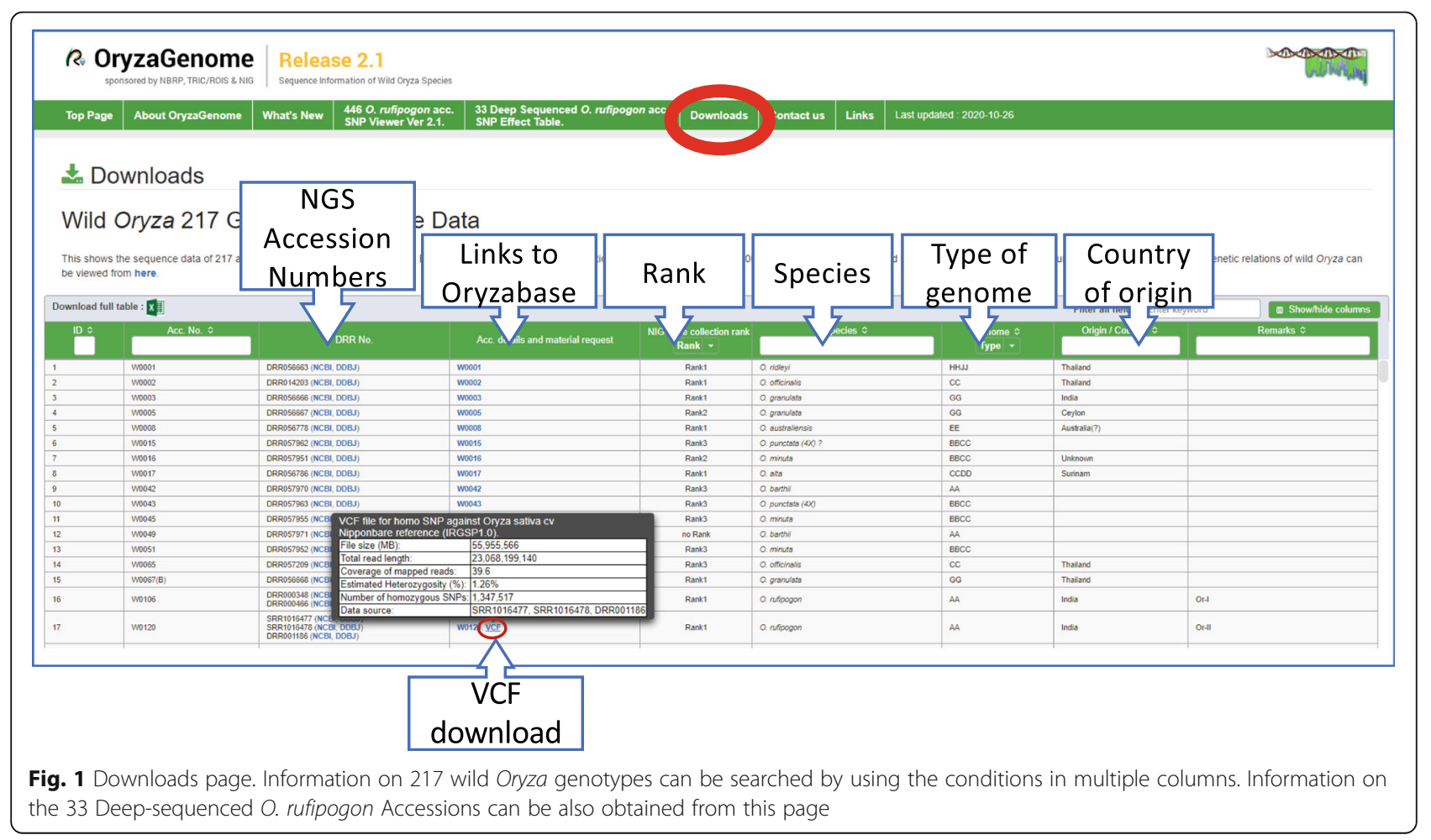


their variants against the japonica reference genome (Kawahara et al. 2013) are available in VCF files (Table 1; Supp. Table 1). OryzaGenome2.1 provides wild Oryza genotype information in five categories: (1) 217 Oryza Genotypes; (2) 33 O. rufipogon Genotypes and Variants; (3) SNP Viewer for 446 Imputed O. rufipogon Genotypess, with new information tracks; (4) SNP Effect Table function to search for the effects of variants in $33 \mathrm{O}$. rufipogon accessions against IRGSP1.0; and (5) Misc Downloads.

\section{Oryza Genotypes}

In the Downloads section of OryzaGenome2.1, nextgeneration sequencing (NGS)-derived genotypes of 217 accessions from 19 wild Oryza species (O. rufipogon, O. barthii, O. longistaminata, O. meridionalis, O. glumaepatula, O. punctata, O. minuta, O. officinalis, O. rhizomatis, $O$. eichingeri, $O$. latifolia, $O$. alta, $O$. grandiglumis, O. australiensis, O. brachyantha, O. granulata, O. meyeriana, O. ridleyi, and O. longiglumis) are provided. The 19 wild species belong to 9 genome types (AA, BB, CC, BBCC, CCDD, EE, FF, GG, and $\mathrm{HHJJ}$ ), representing much more genomic divergence than in the previous version. Each accession has been genotyped by Illumina paired-end short-read DNA sequencing (100to 150 -bp read length for one end) of over $8 \times$ coverage (average $18 \times$ coverage). Users can browse the sequencing platform, total bases, coverage, and file size in a pop-up window by mouse-over action at the accession numbers that are hyperlinked to NCBI and DDBJ on the Downloads page (Fig. 1). The meta-information for these accessions is also available on the Downloads page. Data can be selected and sorted by changing search conditions such as species, genome type, growth habit (annual or perennial), rank in the core collection, and origin (Fig. 1). To obtain phenotype information on each accession, links are provided to web pages in Oryzabase (Kurata and Yamazaki 2006) describing the characteristics of each accession (Fig. 1), as well as to request resources.

Using the genotype information in Oryzagenome2.1, we analyzed the genome diversity of genus Oryza. K-mer analysis was conducted to estimate the size of genome, the content of repetitive sequences and the heterozygosity in the genome of each accession (Supplementary Table 2). It turned out that there is a significant degree of variance in the genome size ranging approximately $250 \sim 1000 \mathrm{Mbp}$ and this is largely concomitant with previous reports (Stein et al., 2018). The size of the genome tends to be similar among the same genome type and among the same species. The genome size of $O$. brachyantha and $O$. ridleyi are the smallest and the largest, respectively (Supplementary Fig. 1A). There is a positive linear correlation between genome size and repeat length among Oryza including diploid and tetraploid species (Supplementary Fig. 1A). However, the content of repetitive sequences in tetraploid species is rather constant, despite the large difference in the genome size (Supplementary Fig. 1B). This suggests that the genome size of ancestral diploid species may have the major impact on that of tetraploid species (Shenton et al., 2020). Overall, genotype data available from Oryzagenome2.1 would contribute to analyze various aspect of genome diversity seen among genus Oryza.

\section{O. rufipogon Genotypes and Variants}

Because O. rufipogon is the progenitor of Asian cultivated rice, $O$. sativa, we paid special attention to it and obtained higher coverage (average 19.5 $\times$ ) for 33 accessions of $O$. rufipogon. The raw sequence data of these accessions, as well as VCF files containing information on variants against Os-Nipponbare-Reference-IRGSP-1.0, are provided on the Downloads page (Fig. 1). Users can browse the coverage, estimated heterozygosity, number of homozygous polymorphisms, and file size in a pop-up window by mouse-over action at the VCF download icons. In addition, the numbers of homozygous SNPs, and small insertions and deletions in 33 O. rufipogon accessions are provided in Supplementary Table 3.

\section{SNP Viewer with New Information Tracks for 446 Imputed O. rufipogon Genotypes}

The genome-browser-oriented SNP Viewer (Fig. 2) for the 446 imputed $O$. rufipogon genotypes (see the Misc Downloads section) is updated in this release. Chromosome coordinates, genome sequences, and gene annotations are based on the latest japonica reference genome, Os-Nipponbare-Reference-IRGSP-1.0 (Kawahara et al. 2013). In addition, this version graphically presents genomic regions where selective sweeps may have occurred during domestication of $O$. sativa from $O$. rufipogon populations (Fig. 2) according to data published in Huang et al. (2012). Genomic regions in O. sativa potentially affected by selective sweep are color-coded: those in the whole population with red bars, those only in indica populations with yellow bars, and those only in japonica populations with green bars. The SNP Viewer also graphically shows regions with higher mutation rates (i.e., highly differentiated loci). The genomic coordinates show regions or loci that appear to have been exposed to particular selective pressures through the process of domestication. In addition to RAP-DB (Sakai et al. 2013) and MSU gene models (Ouyang et al. 2007), shown near the top of the viewer, the SNP Viewer now includes Committee on Gene Symbolization, Nomenclature and Linkage (CGSNL) gene names (McCouch 2008) and can instantly supply biological information. For example, Fig. 2 shows the region around Os05g0187500 encoding GW5 (Shomura et al. 2008), which 


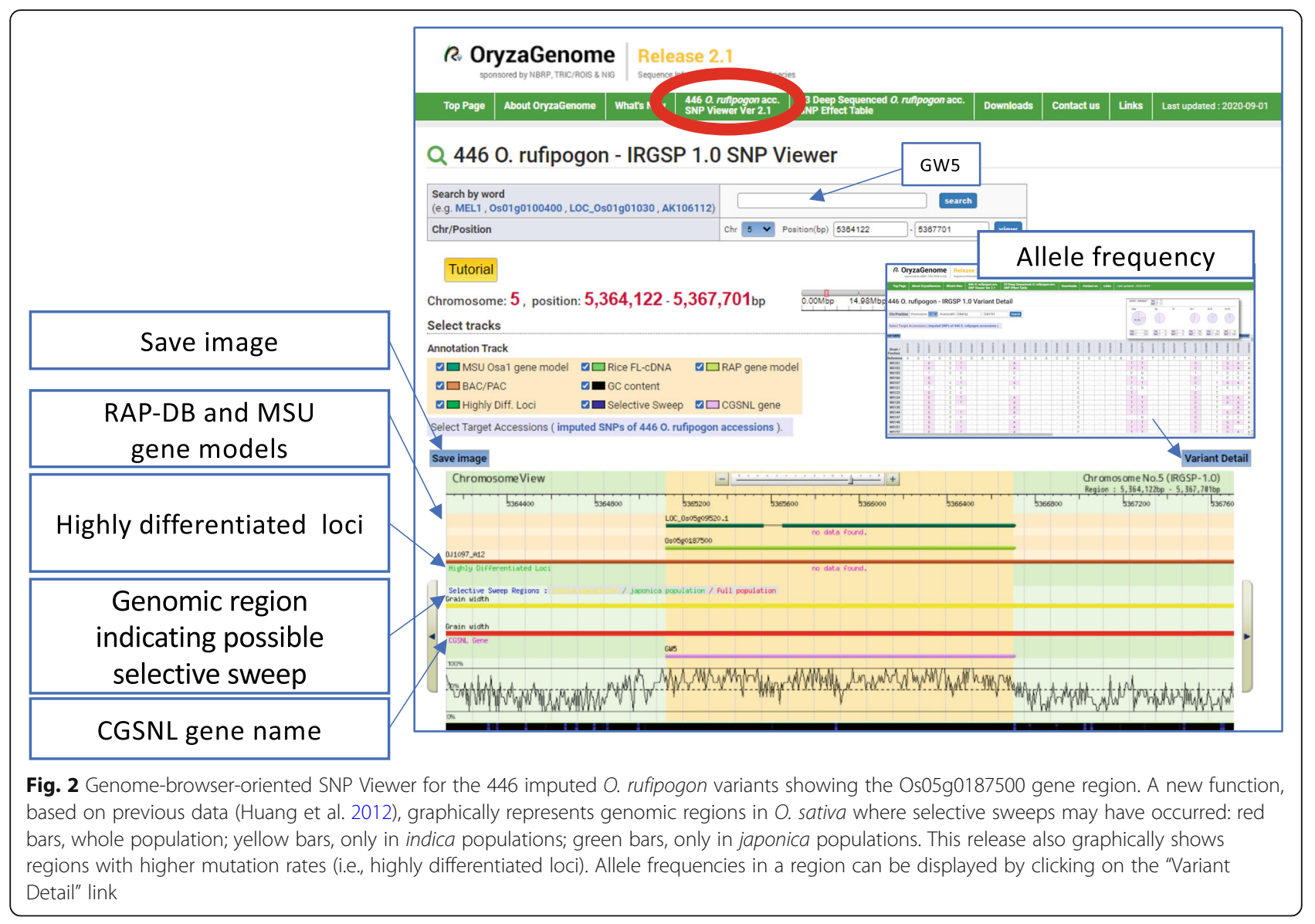

quantitatively controls rice grain width, and indicates that selective pressure might have been applied in this region.

\section{SNP Effect Table Function}

A key update in release 2.1 is a fully customized SNP Effect Table function (Fig. 3), which is designed to search for and identify polymorphisms between the 33 deepsequenced $O$. rufipogon genotypes and IRGSP1.0 that have specific characteristics. Variant information for 33 O. rufipogon accessions (average coverage 19.5x) was obtained by using the SnpEff tool (Cingolani et al. 2012). The SNP Effect Table function provides an output table containing polymorphism information such as position (IRGSP1.0 chromosome coordinate), nearest gene ID, position relative to the nearest gene, type of polymorphism, effect of polymorphism, accessions carrying the polymorphic allele, and so on. SNPs of interest can be searched by gene locus ID, gene symbol synonym, gene name synonym (partial match), or chromosome coordinate (Fig. 3a), and the resultant SNP list is displayed in tabular format (Fig. 3b). The SNP Effect Table can be exported as an Microsoft Excel file. In each entry, the SNP variant type is color-coded, and the position of
SNPs in the region is shown on simple map graphs (Fig. 3b).

\section{Misc Downloads}

In the Misc Downloads section in the Downloads page, miscellaneous information used in or associated with OryzaGenome2.1 is provided. Files for tabular-formatted genotype, VCF, and Plink-formatted genotype of newly imputed SNPs of the 446 O. rufipogon accessions are available from the Misc Downloads section. These genotype files were newly produced for OryzaGenome2.1 and were made by imputation followed by removal of missing data for use in genome-wide association studies (GWAS) (see Materials and Methods). BED-formatted annotations and $F$ st values for the 446 O. rufipogon accessions published in Huang et al. (2012) and used in the SNP Viewer are also available at Misc Downloads.

In OryzaGenome1, an initial set of imputation-derived variant information on 446 O. rufipogon accessions, which contained missing data, was released. This information is still available from the Misc Downloads section of the Downloads page of release 2.1 (use link "Download imputed SNPs of 446 O. rufipogon accessions") as well as information on these 446 accessions 


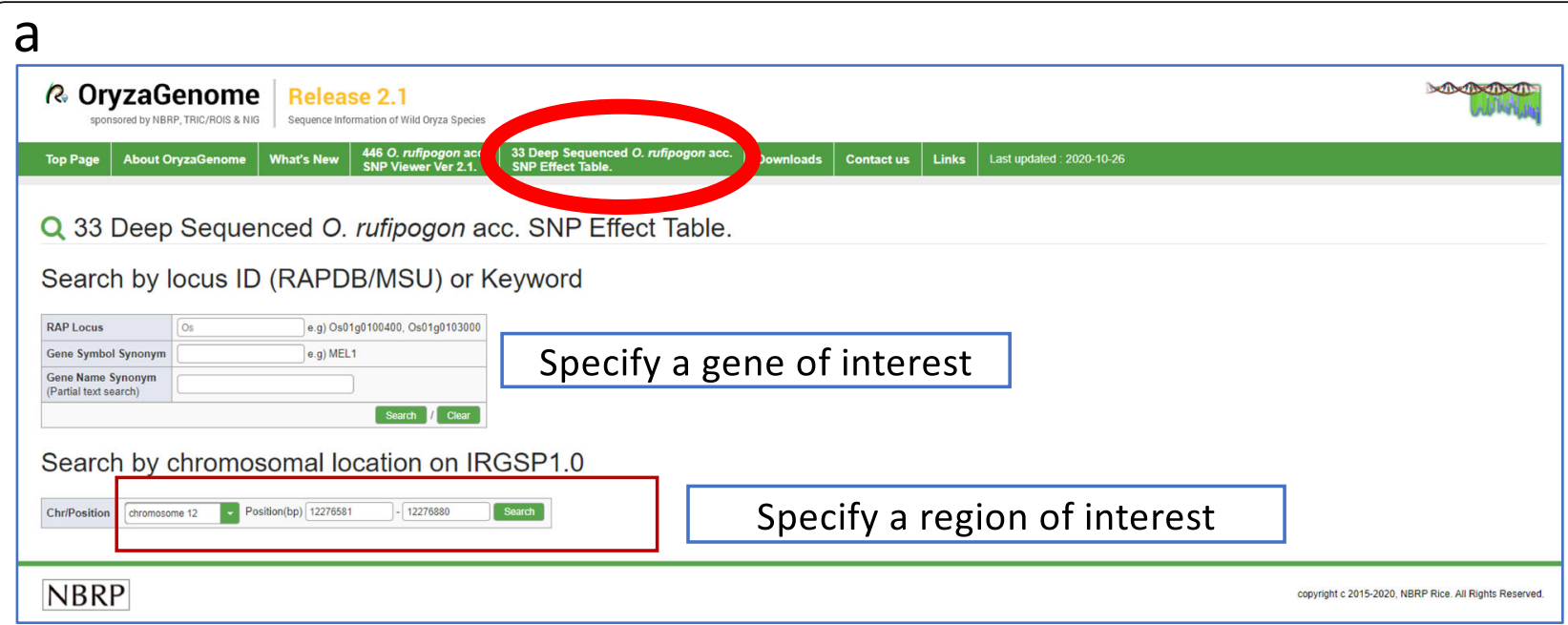

b

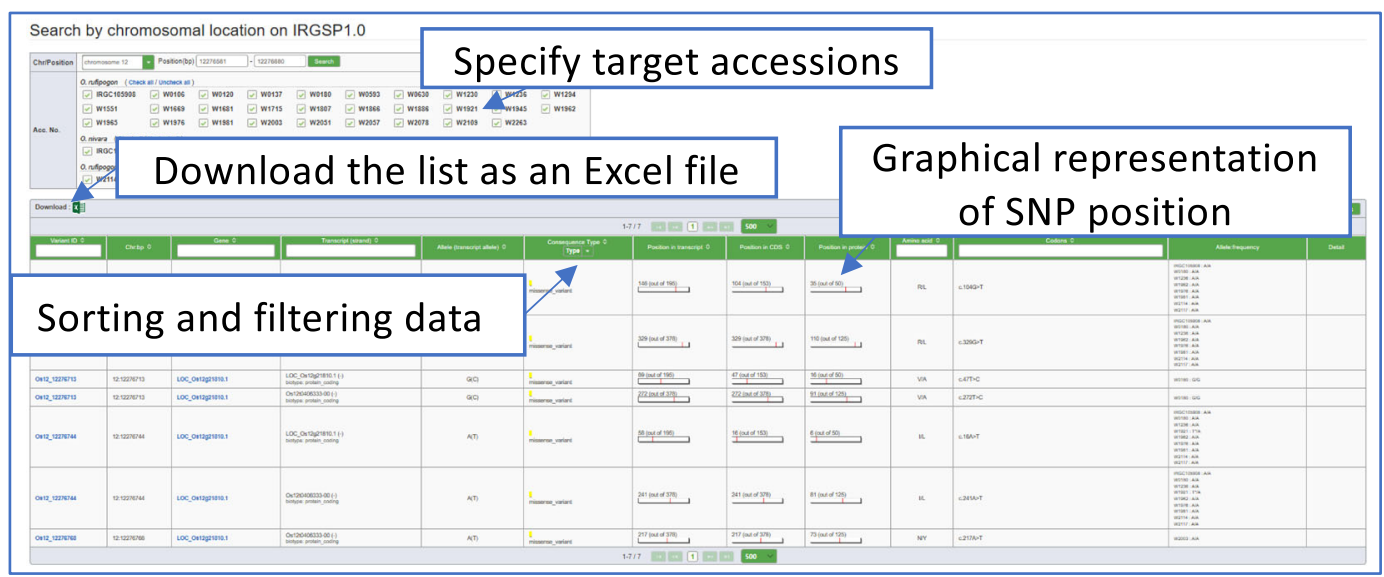

Fig. 3 SNP Effect Table. a A new function allows users to search for SNPs with specific characteristics in the 33 Deep-Sequenced Oryza rufipogon Accessions by gene locus ID or IRGSP1.0 chromosome coordinate, and (b) the resultant SNP list is displayed in tabular format

including accession number, habitat/country of origin, ecotype, sequence coverage, and deposited IDs of NGS raw data.

\section{Conclusions}

The goal of OryzaGenome is to facilitate comparative and evolutionary genomic analysis of diverse wild Oryza accessions. Most of the wild Oryza bioresources used for acquisition of genotype data in OryzaGenome are preserved at the National Institute of Genetics in Japan. These materials were collected during the $1950 \mathrm{~s}$ to 1980 s from around the world, and their biomaterials (such as seeds) are available from Oryzabase (https:// shigen.nig.ac.jp/rice/oryzabase/) upon request. Thus, OryzaGenome will help to promote the use of wild Oryza bioresources in basic research and speed up the identification of critical agricultural-trait-related genes from these bioresources. OryzaGenome aims to promote rice breeding science as well as potential future crop improvement. OryzaGenome2.1 is a significant update, providing a genus-wide comprehensive genomic repository for wild Oryza species.

Improved web-based tools (the SNP Effect Table and SNP Viewer) allow visual inspection of SNPs of interest and the evolutionary background of the surrounding regions in O. rufipogon. For genes with known function, it is now possible to quickly narrow down SNPs linked to regions that may affect their function. Lists of SNPs in candidate regions can be quickly obtained on the web interface and downloaded as an Excel file.

\section{Future Directions}

OryzaGenome2.1 provides access to a wide variety of Oryza genotypes, including newly sequenced genotypes 
of seven tetraploid Oryza species: O. minuta (BBCC), O. punctata (BBCC), O. alta (CCDD), O. grandiglumis (CCDD), O. latifolia (CCDD), O. longiglumis (HHJJ), and O. ridleyi $(\mathrm{HHJJ})$. Compared with other comprehensive databases for Oryza genomics (Alexandrov et al. 2015; Wing et al. 2007), OryzaGenome2.1 includes substantially more diverse genotype information. These diverse rice genotypes will be key resources not only for straightforward evolutionary studies, but also for polyploid genome biology in Oryza (Shenton et al. 2020). Another important feature of OryzaGenome2.1 is that it offers functions to extract polymorphisms between cultivated rice $(O$. sativa) and its wild progenitor (O. rufipogon) and to survey the effect of polymorphisms. These functions will contribute to the analysis of domestication processes and the identification of agriculturally important genes from wild progenitors.

We expect that cross-references between databases storing Oryza genome information, such as OryzaGenome2.1, OMAP (http://www.omap.org/index.html), Gramene (http://www.gramene.org), Ensembl Plants (https://plants.ensembl.org/index.html), and Rice SNP seek (https://snp-seek.irri.org), will greatly facilitate evolutionary studies of Oryza and contribute to agricultural science. We also foresee that installing and integrating genome browsers for resequencing data, such as TASUKE+, in future versions of OryzaGenome will improve the user experience (Kumagai et al. 2019).

\section{Supplementary Information}

The online version contains supplementary material available at https://doi. org/10.1186/s12284-021-00468-x.

Additional file 1: Table S1. Accession list of OryzaGenome2.1.

Additional file 2: Table S2. Summary of $K$-mer analysis using genotypes of 217 wild Oryza accessions.

Additional file 3: Table S3 Number of homozygous small nucleotide polymorphisms in 33 O. rufipogon.

Additional file 4: Fig. S1. Analysis of genome diversity among genus Oryza. A. A plot of estimated genome size and estimated repeat length among genus Oryza. B. A plot of estimated genome size and estimated repeat content among genus Oryza.

\section{Abbreviations}

CGSNL: Committee on Gene Symbolization, Nomenclature and Linkage; DDBJ: DNA Data Bank of Japan; DRA: DDBJ Sequence Read Archive; GWAS: Genome-wide association studies; NGS: Next-generation sequencing; SNP: Single nucleotide polymorphism; VCF: Variant call format

\section{Acknowledgments}

We thank the NBRP and NIG Bioresource Project for supporting the preservation of wild accessions and database construction. Some computations were performed on the NIG supercomputer at ROIS National Institute of Genetics. We acknowledge the support of Gaku Kimura, Hiroki Watanabe, and Rie Tsuchiya for web server management.

\section{Authors' Contributions}

HKK, HO, TE, YT, AO, YS, MYH, KT, TS, and MNT performed the experiments. ZXW, BH, AT, AF, HI, KN, YN, SK NK, and YS conceived and designed the study. All authors read and approved the final manuscript.

\section{Funding}

This work was supported by a Japan Society for the Promotion of Science Grant-in-Aid A for Scientific Research [25252005 to N.K.]; the National BioResource Project (NBRP) [Genome Information Upgrading Program to Y.S., N.K., and A.T.]; and the National Institute of Genetics [NIG-JOINT88B2018 to H.K.K.].

\section{Availability of Data and Materials}

The datasets generated and analyzed during this study are available in OryzaGenome2.1 (http://viewer.shigen.info/oryzagenome21detail/index. xhtml).

Accession numbers of short read sequence data are listed in Supplemental Table 1 and on the OryzaGenome2.1 Downloads page (http://viewer.shigen. info/oryzagenome21detail/downloads/index.xhtml). Genetic variation data of 33 O. rufipogon accessions are deposited to European Variation Archive under accession numbers PRJEB42581 (Project) and ERZ1714350 (Analyses).

\section{Ethics Approval and Consent to Participate}

Not applicable.

\section{Consent for Publication}

Not applicable.

\section{Competing Interests}

No conflicts of interest are declared.

\section{Author details}

${ }^{1}$ Department of Agricultural and Environmental Biology, Graduate School of Agricultural and Life Science, The University of Tokyo, Bunkyo 1-1-1, Tokyo 113-8657, Japan. ${ }^{2}$ King Abdullah University of Science and Technology, Computational Bioscience Research Center, Biological and Environmental Sciences \& Engineering Division, Thuwal 23955-6900, Saudi Arabia. ${ }^{3}$ Dynacom Co., Ltd., World Business Garden, Marive East 25F, 2-6-1, Nakase, Mihama-ku, Chiba-shi, Chiba 261-7125, Japan. ${ }^{4}$ National Institute of Genetics, Yata 1111, Mishima, Shizuoka 411-8540, Japan. ${ }^{5}$ Institute of Crop Science, NARO, Kannondai 2-1-2, Tsukuba, Ibaraki 305-8518, Japan. ${ }^{6}$ RIKEN Center for Sustainable Resource Science, 1-7-22 Suehiro-cho, Tsurumi-ku, Yokohama, Kanagawa 230-0045, Japan. ${ }^{7}$ National Center for Gene Research, Institute of Plant Physiology and Ecology, Shanghai Institutes for Biological Sciences, Chinese Academy of Sciences, 500 Caobao Road, Shanghai, China.

Received: 31 October 2020 Accepted: 17 February 2021

Published online: 04 March 2021

\section{References}

Alexandrov N, Tai S, Wang W et al (2015) SNP-seek database of SNPs derived from 3000 rice genomes. Nucleic Acids Res. https://doi.org/10.1093/nar/gku1 039

Bolger AM, Lohse M, Usadel B (2014) Trimmomatic: a flexible trimmer for Illumina sequence data. Bioinformatics. https://doi.org/10.1093/bioinformatics/btu170

Browning SR, Browning BL (2007) Rapid and accurate haplotype phasing and missing-data inference for whole-genome association studies by use of localized haplotype clustering. Am J Hum Genet 81:1084-1097. https://doi. org/10.1086/521987

Cingolani P, Platts A, Wang LL et al (2012) A program for annotating and predicting the effects of single nucleotide polymorphisms, SnpEff: SNPs in the genome of Drosophila melanogaster strain w1118; iso-2; iso-3. Fly (Austin). https://doi.org/10.4161/fly.19695

Huang X, Kurata N, Wei X et al (2012) A map of rice genome variation reveals the origin of cultivated rice. Nature 490:497-501. https://doi.org/10.1038/na ture11532

Kawahara Y, de la Bastide M, Hamilton JP et al (2013) Improvement of the Oryza sativa Nipponbare reference genome using next generation sequence and optical map data. Rice 6:4. https://doi.org/10.1186/1939-8433-6-4 
Kumagai M, Nishikawa D, Kawahara Y, Wakimoto H, Itoh R, Tabei N, Tanaka T, Itoh T (2019) (2019) TASUKE+: a web-based platform for exploring GWAS results and large-scale resequencing data. DNA Res 26(6):445-452

Kurata N, Yamazaki Y (2006) Oryzabase. An integrated biological and genome information database for rice. Plant Physiol. https://doi.org/10.1104/pp.105. 063008

Li H, Durbin R (2010) Fast and accurate long-read alignment with burrowswheeler transform. Bioinformatics 26:589-595. https://doi.org/10.1093/ bioinformatics/btp698

Marcais G, Kingsford C (2011) A fast, lock-free approach for efficient parallel counting of occurrences of k-mers. Bioinformatics 27:764-770. https://doi. org/10.1093/bioinformatics/btr011

McCouch SR (2008) Gene nomenclature system for rice. Rice. 1:72-84. https://doi. org/10.1007/s12284-008-9004-9

McKenna A, Hanna M, Banks E et al (2010) The genome analysis toolkit: a MapReduce framework for analyzing next-generation DNA sequencing data. Genome Res 20:1297-1303. https://doi.org/10.1101/gr.107524.110

MSU Rice Genome Annotation Project Team (2015) Rice genome annotation project. Http://RicePlantbiologyMsuEdu/

Nonomura K-I, Morishima H, Miyabayashi T et al (2010) The wild Oryza collection in national BioResource project (NBRP) of Japan: history, biodiversity and utility. Breed Sci 60:502-508. https://doi.org/10.1270/jsbbs.60.502

Ohyanagi H, Ebata T, Huang X et al (2016) OryzaGenome: genome diversity database of wild oryza species. Plant Cell Physiol 57:e1. https://doi.org/10.1 093/pcp/pcv171

Ouyang S, Zhu W, Hamilton J et al (2007) The TIGR Rice genome annotation Resource: Improvements and new features. Nucleic Acids Res. https://doi. org/10.1093/nar/gkl976

Sakai H, Lee SS, Tanaka T et al (2013) Rice annotation project database (RAP-DB): An integrative and interactive database for rice genomics. Plant Cell Physiol. https://doi.org/10.1093/pcp/pcs183

Shenton M, Kobayashi M, Terashima S et al (2020) Evolution and diversity of the wild rice Oryza officinalis complex, across continents genome types, and ploidy levels. Genome Biol Evol. https://doi.org/10.1093/gbe/evaa037

Shomura A, lzawa T, Ebana K et al (2008) Deletion in a gene associated with grain size increased yields during rice domestication. Nat Genet 40:10231028. https://doi.org/10.1038/ng.169

Stein JC, Yu Y, Copetti D, Zwickl DJ, Zhang L, Zhang C, Chougule K, Gao D, Iwata A, Goicoechea JL et al (2018) Genomes of 13 domesticated and wild rice relatives highlight genetic conservation, turnover and innovation across the genus Oryza. Nat Genet 50:285-296

Vurture GW, Sedlazeck FJ, Nattestad M, Underwood CJ, Fang H, Gurtowski J, Schatz MC (2017) GenomeScope: fast reference-free genome profiling from short reads. Bioinformatics 33:2202-2204. https://doi.org/10.1093/bioinforma tics/btx153

Wang W, Mauleon R, Hu Z et al (2018) Genomic variation in 3,010 diverse accessions of Asian cultivated rice. Nature. https://doi.org/10.1038/s41586-01 8-0063-9

Wing RA et al (2007) The Oryza map alignment project (OMAP): a new resource for comparative genome studies within Oryza. In: Rice functional genomics. Springer, New York, NY, pp pp395-pp409. https://doi.org/10.1007/0-387-4 8914-2_15

\section{Publisher's Note}

Springer Nature remains neutral with regard to jurisdictional claims in published maps and institutional affiliations.

\section{Submit your manuscript to a SpringerOpen ${ }^{\circ}$ journal and benefit from:}

- Convenient online submission

- Rigorous peer review

- Open access: articles freely available online

- High visibility within the field

- Retaining the copyright to your article

Submit your next manuscript at $\boldsymbol{\nabla}$ springeropen.com 